\title{
Research on multi-sources knowledge fusion model for industrial
}

\section{innovative design}

\author{
Wei Liu ${ }^{1, a}$, Shuai Liu ${ }^{1, b}$, Limeng Liu ${ }^{1, c}$, Runhua $\operatorname{Tan}^{1, d}$ \\ ${ }^{1}$ School of Mechanical Engineering , Hebei University of technology, Tianjin 300130, China; \\ alwofhebut@126.com, ${ }^{\mathrm{b}}$ Isfree@163.com, ${ }^{\mathrm{C}}$ liulm3@163.com, ${ }^{\mathrm{d}}$ rhtan@hebut.edu.cn
}

Keywords: Multi-sources knowledge ,knowledge fusion, industrial innovative design

\begin{abstract}
This paper proposes a new model to describe the multi-sources knowledge fusion, which works as an effective method to generate the innovative results for industrial design. Firstly, we analyze the structure of knowledge fusion in industrial design. Then we find that the process of industrial design is driven by multi-sources from various knowledge background. Subsequently, we build a multi-sources knowledge fusion design workflow model to assist the practical industrial design and to improve its efficiency. Lastly, we use a new kind of bionic helmet to verify the proposed method.
\end{abstract}

\section{Introduction}

In knowledge economy society, how to design a competitive industrial product has become an essential issue for companies to seize the development chances especially for the manufactures[1]. It has been proved that the knowledge has been a key to the success for generating innovative ideas[2]. There is a growing fact that the design for industrial products needs more and more knowledge from multiple disciplines' background[3]. Biological inspired design has put out beside technological knowledge biological knowledge also works as an important factor for trigger innovative industrial design[4]. Theory of innovation driven by design presented a even wider viewpoints on product innovation which needs the knowledge from psychology and sociology[5]. Though the importance of multi-sources knowledge in modern industrial design has been stressed repeatedly, which is still needs further study on how to express the multi-sources knowledge related to industrial design as well as how to make best of various originated knowledge. Therefore, in this paper, we construct a fusion model of multi-sources knowledge for industrial products and their design jobs, then a design process based on the proposed models try to improve the efficiency of industrial innovation driven by multi-sources knowledge.

\section{2 . Multi-sources knowledge fusion model in industrial product}

To analyze the composition of factors in industrial design, we proposed a two-circle fusion model shown in Fig 1to express the multi-sources knowledge which determine the industrial design. From the Fig 1, the inner circle of model is made up of elements which affects design directly, such as the physical parameters, appearance features, functions, structure and man-machine interfaces and so on , all these can determine the patterns, functions and even the marketing value of the industrial products directly. While the outer circle of the model mainly contains factors can influence the inner circle elements as well as the attributes of industrial products, these factors 
include natural science disciplines such as physics, material science, processing technology, electronics and information science and the social sciences such as psychology, aesthetics, behavioral science, marketing management, economics and even the ethnics.

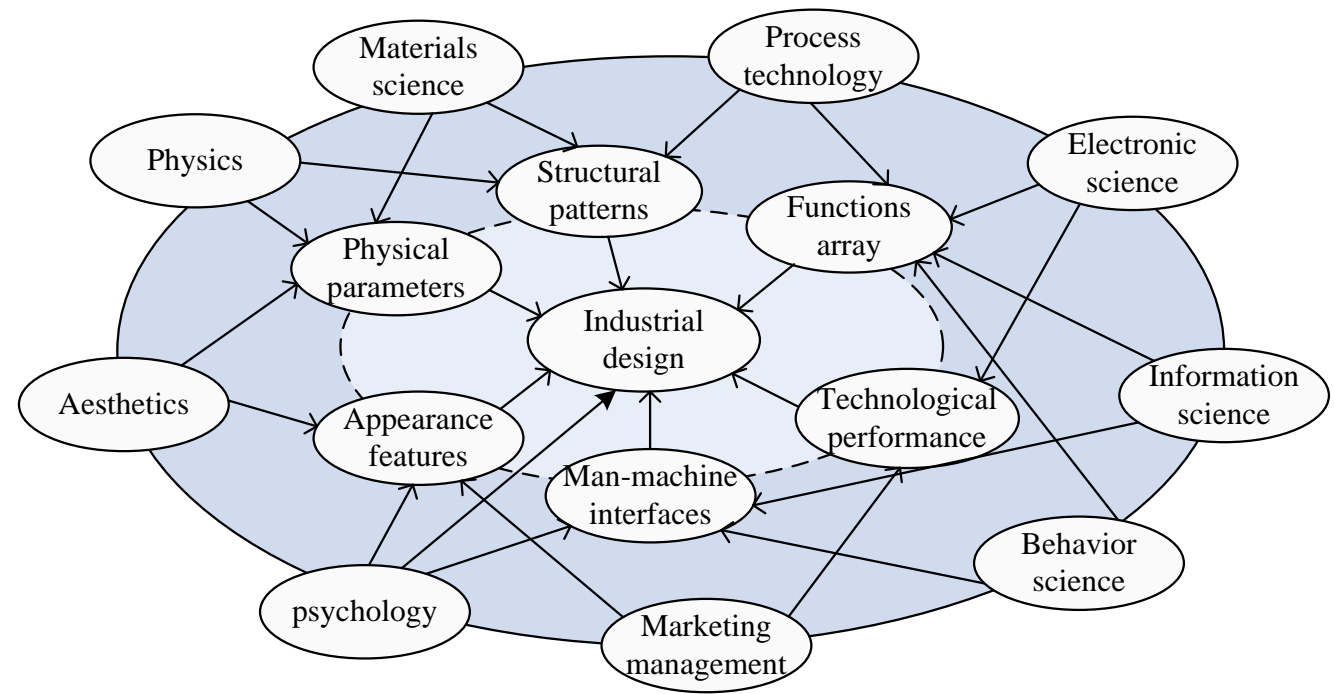

Fig. 1 Two or more references

From what discussed above, the innovative design for a successful industrial product needs a wide range knowledge pool, how to handle such abundance resource resources and get the innovation result become an important issue needs further research.

\section{3 . Process by multi-sources knowledge for industrial product innovation}

The classical innovation process model has put the new product design into three steps: fuzzy front ends(FFE), new product develop (NPD) and marketing. The design for industrial products driven by multi-sources knowledge also accord with the model shown in Fig2, where an average successful product needs about 3000 ideas in stage of FFE and 14 prototypes under development in NPD. To increase the number of innovative ideas in FFE seems a possible way to improve the chance of industrial products innovation, therefore, try to broad the range of knowledge can inspire the innovative ideas is a feasible way to bring up the industrial innovation. In this paper, we build a knowledge fusion resources database which contains customers back feed as well as marketing analysis information, and successful design cases, even the principles or prototypes from biology, we put these knowledge all together to inspire the innovative ideas in FFE. Compared with the traditional method which just relay on the company itself market and customers analysis, the new fusion model can make use of methods from the design by analogy and bionics to inspire more innovative ideas obviously.

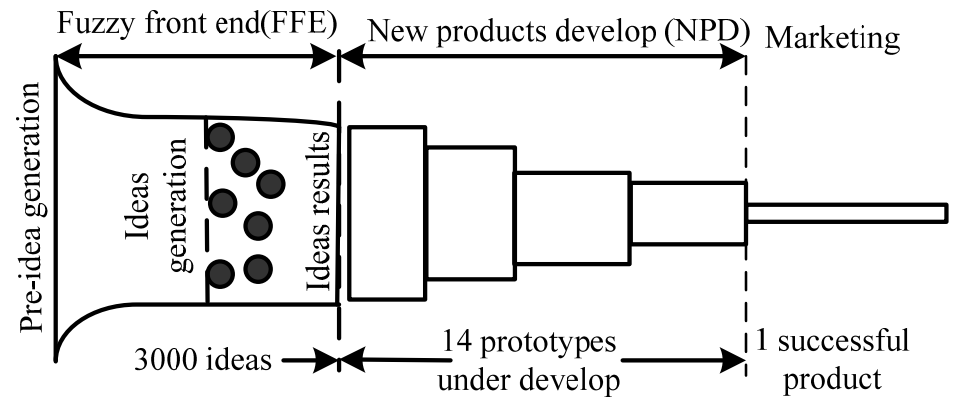

Fig. 2 Process model of new products development 
After the FFE stage the next step is to put the knowledge from multidiscipline background together to achieve the industrial design goals. To infuse the various kinds of knowledge in product design, we propose a representing method based on similarity of flows and attributes. We redefine the targets of industrial products' design as change the input flow into the output one, such as we define the function of heater as the change the electric current (input) into heat(output). By this means we can extract the useful principles from different fields to embed into the known product structure and turn it into a new one. With the help of similarity judge method, we can even get the most promising prototype from existing design cases or biological ones to get a better industrial innovative design result.

The whole workflow of design process driven by multi-sources knowledge for industrial innovative design is shown in Fig 3, which includes three main steps: the first step is multi-sources inspiring knowledge fusion for FFE, the second one is combination method based on flow similarity and attributes, the third step is verification method based on similarity judgment and other value engineering methods to help get the most appropriate design result.

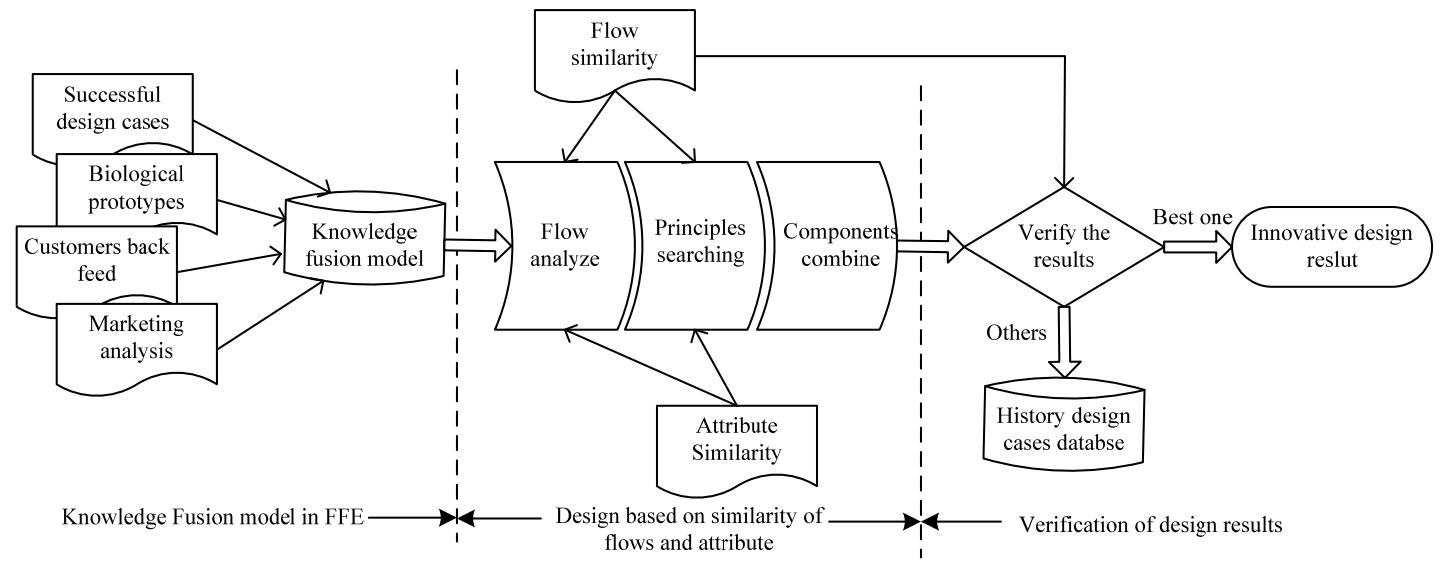

Fig. 3

workflow of industrial innovation driven by multi-sources knowledge

\section{Case study and conclusion}

With the model and process proposed above, we design a new kind of crash helmet. Firstly, we use the keywords " shock proof" to search the patents database as well as the biological knowledge, then we find the skull of woodpecker(shown in Fig 4(a)) is promising prototype for this design task. The second step is to analyze the flows and the components, we drew the described figure of the biological system of skull of woodpecker in Fig 4(b), with the described figure for the structure of woodpecker, we can find the technical counterparts to every element in its structure according to flows and attributes' similarity. The design result of the new crash helmet is shown in Fig 4(c). 

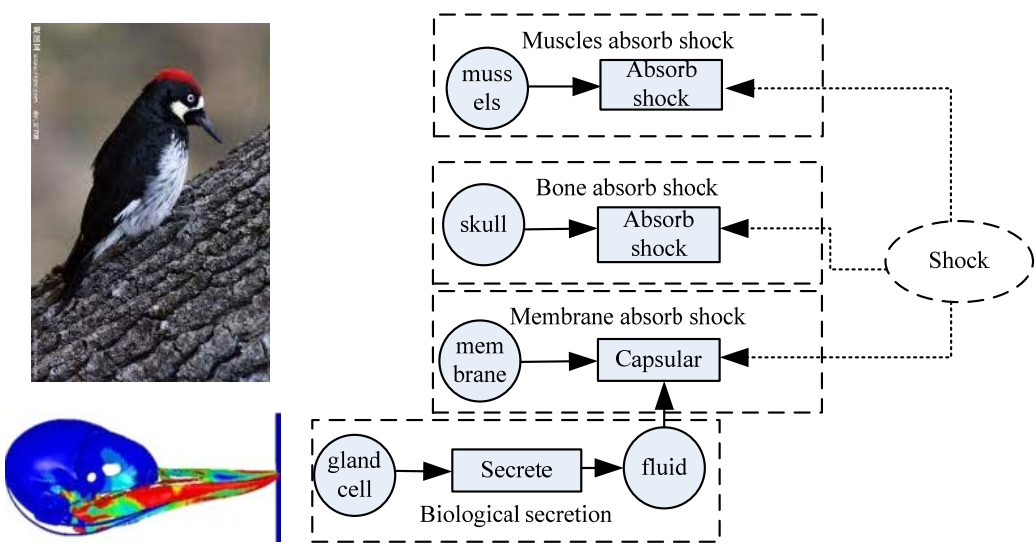

(a)

(b)

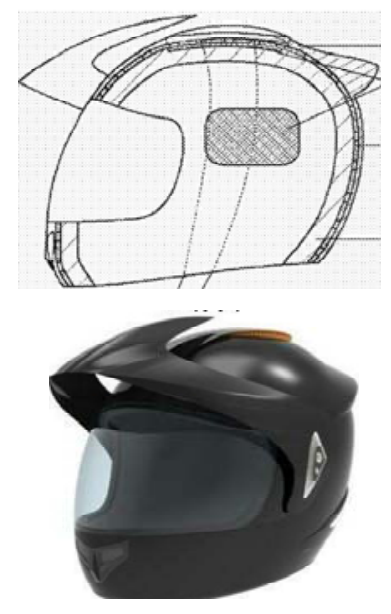

(c)

Fig 4. A new crash helmet design driven by proposed workflow

Compared with the existing industrial products, the design result has better performance both in technological and in appearance features, which means a higher ability in marketing competition.

\section{5 . Acknowledgement}

This paper is sponsored by China natural foundation( grand No. 51675159) and Graduate Innovation fund of Hebei province China ( grand No. 220056).

\section{References}

[1] Chesbrough, H.W. The era of open innovation, MIT Sloan Management Review, 44 (2003) 35-41.

[2] Robert. N, Katarzyna, B . Innovations within knowledge management, Journal of Business Research 69 (2016) 1577-1581.

[3] March, J.G., Exploration and exploitation in organizational learning, Organ. Sci, 2(1991)71-87.

[4] Cooley M, Architecture or bee?, London: The Hogarph Pree,1987.

[5] Robert. N, Katarzyna, B . Innovations within knowledge management, Journal of Business Research 69 (2016) 1577-1581. 